\title{
Perspectiva sensorial de uma nova formulação de requeijão cremoso: um estudo de
}

\section{caso}

Sensory perspective of a new cream cheese formulation: a case study

Perspectiva sensorial de una nueva formulación de queso crema: un estudio de caso

\begin{abstract}
Resumo
A análise sensorial por interpretar e medir as reações às características dos alimentos auxilia na avaliação de percepção sobre os produtos e sua possível aceitação pelo mercado consumidor. O presente artigo é o resultado de um estudo de caso referente a perspectiva dos funcionários de um laticínio em relação a nova formulação do requeijão cremoso. A análise sensorial foi realizada com 30 avaliadores não treinados, utilizando os testes de aceitação por escala hedônica de nove pontos para os atributos textura, sabor, aparência e aroma e a intenção de compra por meio de uma escala hedônica de cinco pontos. Além disso, foi aplicado um formulário composto por sete questões de múltipla escolha, referente ao sexo, idade, nível de escolaridade, renda, frequência que consome o produto, forma com que consome, quais os atributos que lhe fazem gostar de requeijão cremoso, e por fim quais os atributos que o consumidor costuma analisar em um produto no momento da compra, visando conhecer melhor o perfil dos consumidores em potencial. A análise de dados utilizada foi a estatística descritiva, realizada através do software Excel 2013. O produto teve excelente aceitação dos atributos sensoriais e mais de $80 \%$ dos provadores certamente comprariam o produto. Concluiu-se neste estudo que a reformulação do requeijão é viável e identificou-se que um perfil de consumidor em potencial podem ser as mulheres, na faixa etária de 22 a 40 anos, com ensino superior completo e renda de dois a quatro salários mínimos.
\end{abstract}

Palavras-chave: Consumo; Derivados lácteos; Escala hedônica; Concentrado proteico.

\begin{abstract}
Sensory analysis, by interpreting and measuring reactions to food characteristics, helps in evaluating the perception of products and their possible acceptance by the consumer market. This article is the result of a case study regarding the perspective of the employees of a dairy about the new formulation of cream cheese. Sensory analysis was performed with 30 untrained evaluators, using acceptance tests by a nine-point hedonic scale for texture, flavor, appearance, and aroma attributes and purchase intention through a five-point hedonic scale. In addition, a form consisting of seven multiple-choice questions was applied, referring to sex, age, education level, income, frequency of consuming the product, how it consumes, which attributes make you like cream cheese, and finally, which attributes the consumer usually analyzes in a product at the time of purchase, to better understand the profile of potential consumers. The data analysis used was descriptive statistics, performed using Excel 2013 software. The product had excellent acceptance of sensory attributes and more than $80 \%$ of the panelists would certainly buy the product. It was concluded in this study that the reformulation of cream cheese is feasible and it was identified that a potential consumer profile can be women, aged between 22 and 40 years, with higher education and income of two to four minimum wages.
\end{abstract}

Keywords: Consumption; Dairy derivatives; Hedonic scale; Protein concentrate.

\section{Resumen}

El análisis sensorial, al interpretar y medir las reacciones a las características de los alimentos, ayuda a evaluar la percepción de los productos y su posible aceptación por parte del mercado consumidor. Este artículo es el resultado de un estudio de caso sobre la perspectiva de los empleados de una quesería en relación a la nueva formulación de queso crema. El análisis sensorial se realizó con 30 evaluadores no entrenados, utilizando pruebas de aceptación mediante una escala hedónica de nueve puntos para atributos de textura, sabor, apariencia y aroma e intención de compra mediante una escala hedónica de cinco puntos. Además, se aplicó un formulario compuesto por siete preguntas de opción múltiple, referentes al sexo, edad, nivel educativo, ingreso, frecuencia de consumo del producto, forma en que se consume, qué atributos hacen que le guste el queso crema y, por último, cuáles son los atributos que el consumidor 
suele analizar en un producto en el momento de la compra, con el fin de conocer mejor el perfil de los consumidores potenciales. El análisis de datos utilizado fue estadístico descriptivo, realizado con el software Excel 2013. El producto tuvo una excelente aceptación de los atributos sensoriales y más del $80 \%$ de los panelistas seguramente comprarían el producto. Se concluyó en este estudio que la reformulación del requeijão es factible y se identificó que un perfil de consumidor potencial puede ser mujer, con edad entre 22 y 40 años, con educación superior e ingresos de dos a cuatro salarios mínimos.

Palabras clave: Consumo; Derivados lácteos; Escala hedónica; Concentrado de proteínas.

\section{Introdução}

Entre os anos de 2010 e 2014 a produção de requeijão apresentou crescimento contínuo. Dados levantados por Zarchenco et al. (2017) na publicação Brasil Dairy Trends 2020 apontam o requeijão liderando o ranking como o principal queijo no mercado de commodities do país, além de ser muito consumido pela população de forma geral. Ademais, baseado em dados do IBGE, Siqueira \& Schettino (2021) afirmam que o requeijão é o queijo mais consumido (per capita) na região Sudeste, principalmente nos estados do Rio de Janeiro, São Paulo e Minas Gerais. A região Sul, onde encontra-se o Rio Grande Sul, é a segunda região que mais consome o produto, segundo os autores.

Contudo, atualmente, tem-se estudado sobre mudanças físico-químicas, bioquímicas, microbiológicas e reológicas da fusão do requeijão cremoso, bem como de queijos fundidos, visando a aplicação de novas tecnologias de fabricação e no processo desse importante produto (Van Dender, 2014). A reestruturação das formulações é uma área bastante desafiadora e ao mesmo tempo promissora, pois busca alterar e substituir ingredientes e nutrientes-alvos mantendo sua qualidade, textura, sabor e tempo de vida útil, de modo que essas mudanças não sejam percebidas pelo consumidor final e nem alterem significativamente os custos de produção.

Frente a este cenário, ingredientes promissores são os concentrados proteicos a base de soro de leite. A utilização das proteínas do soro de leite no desenvolvimento de novos produtos e tecnologias alimentares é vista por Maia et al. (2020) como uma grande oportunidade, pois apresenta propriedades funcionais, tecnológicas e nutricionais que acabam despertando o interesse das indústrias alimentícias. Atualmente as proteínas do soro são amplamente utilizadas como ingredientes/matériaprima na produção de novos alimentos, principalmente quando se refere aos lácteos, envolvendo a aplicação de processos, tecnologias, e equipamentos cada vez mais inovadores, como forma de reaproveitar esse subproduto tão importante e benéfico (Bald, 2014). Além disso, a grande solubilidade do soro de leite em uma ampla faixa de pH permite que os concentrados proteicos do soro sejam utilizados em bebidas esportivas. Já a formação de gel, é devido a sua grande capacidade de absorção de água, conferindo-lhes o uso desse ingrediente em carnes processadas, bem como em produtos assados. Os concentrados proteicos de soro podem também ser empregados em bebidas nutricionais, cafés, sopas, e saladas, tudo isso, devido às suas características em emulsificação (Maia et al., 2020).

Alguns estudos testando a substituição de ingredientes por concentrados proteicos já foram relatados, como é o caso do estudo de Bellarde (2005) com doce de leite pastoso, o estudo de Marini (2020) com iogurte e com o próprio requeijão no estudo de Lübeck (2005). Em requeijão cremoso, especificamente, o que se tem empregado, comumente, é a ultrafiltração com teores de gorduras reduzidos, onde a parte da gordura é substituída por concentrados proteicos do soro (Van Dender, 2014). Alguns trabalhos que utilizaram da ultrafiltração acompanhada da substituição da gordura por concentrados proteicos de soro demonstraram que a utilização de concentrado proteico de soro de queijo ultrafiltrado em requeijão cremoso é uma técnica economicamente viável, pois melhora o rendimento (Hoffmann, 2003) além disso o produto teve boa aceitação quanto a cremosidade, sabor, espalhabilidade, cor e firmeza (Gigante, 1998).

Dessa forma, o objetivo do trabalho foi realizar um estudo de caso referente a perspectiva dos funcionários de um laticínio em relação a nova formulação do requeijão cremoso. 


\section{Metodologia}

O trabalho desenvolvido caracterizou-se como sendo uma pesquisa de abordagem quantitativa, pois procurou-se explicar e prever os fenômenos pesquisados, buscando regularidades e relações causais entre elementos (Sampieri et al., 2013), ao mesmo tempo que permitiu a coleta de dados quantificáveis. Além disso, teve carácter descritivo pois pretendeu-se descrever os fatos e fenômenos de determinada realidade (Triviños, 1987), bem como procurou descobrir, com a precisão possível, a frequência com que um fenômeno ocorre, sua relação e conexão com outros, sua natureza e características (Cervo et al., 2012). E, foi de cunho exploratória pois preocupou-se em identificar os fatores que determinam ou que contribuem para a ocorrência dos fenômenos (Gil, 2021).

Quanto aos procedimentos e técnicas adotados, utilizou-se do estudo de caso, aplicação de formulário e análise sensorial, a fim de se obter a percepção dos possíveis consumidores frente a nova formulação de requeijão proposta. Um estudo de caso pode ser caracterizado como um estudo de uma entidade bem definida que visa conhecer em profundidade o como e o porquê de uma determinada situação que se supõe ser única em muitos aspectos ocorre, procurando descobrir o que há nela de essencial e característico (Fonseca, 2002).

\subsection{Amostras de requeijão}

O requeijão cremoso utilizado no presente estudo foi cedido por um laticínio localizado na região Noroeste do Estado do Rio Grande do Sul. O produto em questão sofreu uma reformulação, onde 15\% da massa láctea tradicional foi substituída por um ingrediente proteico a base de proteínas do soro e do leite, próprio para fabricação de requeijão.

Por tratar-se de um produto inovador, a empresa optou por preservar demais informações em relação a formulação utilizada.

\subsection{Avaliação sensorial}

O estudo foi realizado com os funcionários de um laticínio do noroeste do Estado do Rio Grande do Sul, sendo que a população foi composta por 30 avaliadores não treinados, de diferentes idades e gêneros. Para o cálculo do tamanho da amostra adotou-se um erro de $10 \%$ e um nível de confiança de $85 \%$. A equação 1 foi utilizada para a definição da amostra e foi descrita por Santos (2017).

$$
\mathrm{n}=\frac{\mathrm{N} \cdot \mathrm{Z}^{2} \cdot \mathrm{p} \cdot(1-\mathrm{p})}{\mathrm{z}^{2} \cdot \mathrm{p} \cdot(1-\mathrm{p})+\mathrm{e}^{2} \cdot(\mathrm{N}-1)} \quad \text { (Equação 1) }
$$

Cabe ressaltar que foi realizado um recrutamento dos avaliadores, selecionando apenas pessoas com interesse em participar do estudo, que apresentavam disponibilidade de tempo, afinidade pelo produto e sem restrições alimentares aos componentes da formulação da amostra. Também ressalta-se que todos os avaliadores assinaram a um Termo de Consentimento Livre e Esclarecido onde constavam informações sobre a formulação do requeijão e sobre as intenções da pesquisa. A participação foi voluntária e os dados foram mantidos confidenciais.

A avaliação sensorial foi realizada seguindo as instruções de Dutcosky (2015). Cada avaliador recebeu 15 gramas da amostra em um recipiente descartável com colher acompanhados de água e bolachas de água e sal, para limpeza do paladar, a ficha teste e o formulário de pesquisa de perfil de consumidor em potencial.

Os provadores avaliaram o produto por teste de escala hedônica estruturada de nove pontos ( 9 = gostei muitíssimo, $5=$ não gostei nem desgostei e 1 = desgostei muitíssimo), que solicitava o quanto gostou do produto apresentado. Também através desta escala hedônica foram avaliados os atributos de aparência, aroma, textura e sabor. Outra questão pesquisada dizia respeito a intenção de compra, na qual o avaliador, por meio de uma escala de cinco pontos $(5=$ certamente compraria, $3=$ 
talvez sim, talvez não compraria, 1 = certamente não compraria) expressava sua intenção frente ao produto. A última pergunta que compunha a ficha teste era descritiva, onde o provador poderia realizar algum comentário ou consideração a respeito da amostra avaliada. A resposta desta questão, em particular, era opcional.

O formulário de pesquisa de perfil de consumidor em potencial foi composto por sete questões de múltipla escolha, referente ao sexo, idade, nível de escolaridade, renda, frequência que consome o produto, a forma com que consome, quais os atributos que lhe fazem gostar de requeijão cremoso, e por fim quais os atributos que o consumidor costuma analisar em um produto no momento da compra.

\subsection{Análise estatística}

Os dados foram compilados e analisados através de estatística descritiva que foi realizada no Microsoft Excel, versão 2013.

\section{Resultados e Discussão}

De acordo com Dutcosky (2015) a análise sensorial é essencial para avaliar e entender de que forma as alterações na composição podem interferir na cor, sabor, textura e aroma do produto, e como estas são percebidas por cada um dos consumidores. Além disso, o autor explica que as características sensoriais determinam quanto à qualidade e quais aspectos refletem diretamente na rejeição ou aceitação do alimento avaliado por parte dos consumidores. Por isso, é importante identificar todos os atributos desejáveis para que atenda as expectativas dos consumidores e também para que supere os produtos concorrentes.

A análise sensorial realizada contou com uma amostra aleatória de provadores, composta de 30 pessoas adultas não treinadas, sendo na sua maioria mulheres $(53,13 \%)$, com idade entre 22 a 30 anos $(28,13 \%)$ e com nível de escolaridade alto (34,38\% possuem ensino superior completo). Ainda, $46,88 \%$ possuem renda entre dois a quatro salários mínimos (Tabela 1). 
Tabela 1 - Caracterização sociodemográfica da população de estudo.

\begin{tabular}{|c|c|c|}
\hline Variáveis & $\mathbf{N}$ & $\%$ \\
\hline \multicolumn{3}{|l|}{ Sexo } \\
\hline Masculino & 15 & 46,88 \\
\hline Feminino & 17 & 53,13 \\
\hline \multicolumn{3}{|l|}{ Faixa Etária } \\
\hline Até 21 anos & 2 & $6,25 \%$ \\
\hline De 22 a 30 anos & 9 & $28,13 \%$ \\
\hline De 31 a 40 anos & 7 & $21,88 \%$ \\
\hline De 41 a 50 anos & 8 & $25,00 \%$ \\
\hline Mais de 51 anos & 6 & $18,75 \%$ \\
\hline \multicolumn{3}{|l|}{ Escolaridade } \\
\hline Ensino Fundamental Incompleto & 6 & $18,75 \%$ \\
\hline Ensino Fundamental Completo & 2 & $6,25 \%$ \\
\hline Ensino Médio Incompleto & 2 & $6,25 \%$ \\
\hline Ensino Médio Completo & 7 & $21,88 \%$ \\
\hline Ensino Superior Incompleto & 3 & $9,38 \%$ \\
\hline Ensino Superior Completo & 11 & $34,38 \%$ \\
\hline Pós Graduação & 1 & $3,13 \%$ \\
\hline \multicolumn{3}{|l|}{ Renda } \\
\hline Até R\$1.045,00 (Até 1 s.m.) & 1 & $3,13 \%$ \\
\hline De $\mathrm{R} \$ 1.045,00$ (1 s.m.) a $\mathrm{R} \$ 2.090,00$ (1 a 2 s.m.) & 11 & $34,38 \%$ \\
\hline De $\mathrm{R} \$ 2.090,01$ (2 s.m.) a $\mathrm{R} \$ 4.180,00$ (2 a 4 s.m.) & 15 & $46,88 \%$ \\
\hline Mais de $\mathrm{R} \$ 4.180,01$ (mais de 4 s.m.) & 5 & $15,63 \%$ \\
\hline
\end{tabular}

Legenda: $\mathrm{N}$ = número de participantes do estudo. Fonte: Autores.

Quando questionados sobre a frequência de consumo, mais de 53\% responderam que consomem esporadicamente e apenas 6,25\% afirma consumir diariamente (Figura 1).

Figura 1 - Frequência de consumo de requeijão dos provadores.

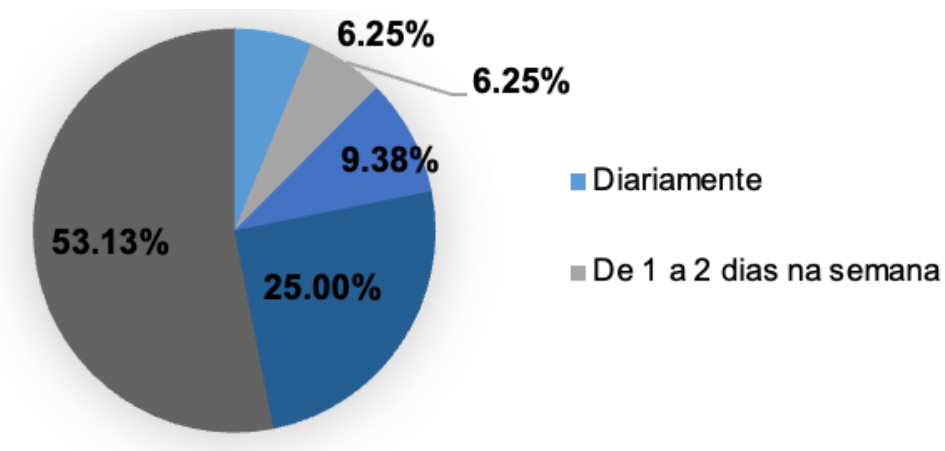

Fonte: Autores.

Sobre a questão forma de consumo, mais de 72,50\% disseram que consomem o requeijão em receitas culinárias, como em pizzas, lasanhas, molhos. O consumo em pães e bolachas foi de $27,50 \%$ e a opção de consumo "somente requeijão sem acompanhamentos" não foi escolhida (Figura 2). 
Figura 2 - Frequência relativa a forma de consumo do requeijão.

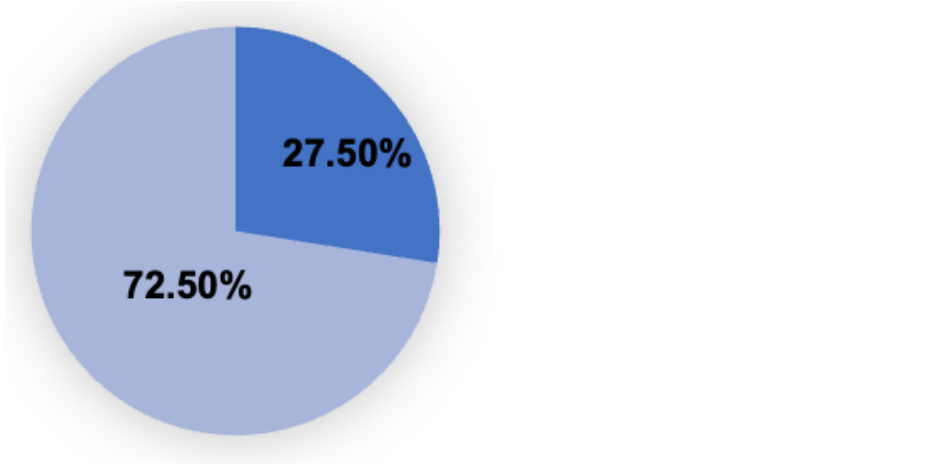

Fonte: Autores.

Segundo Dutcosky (2015) algumas variáveis podem interferir diretamente nos resultados e na escolha do produto, como é o caso das variáveis psicográficas (alimentação, mídias, família, trabalho, esporte, opiniões culturais, sociais, futuro e negócio) e as variáveis demográficas (gênero, idade, classe social, estrutura familiar, educação, etnia, raça, segmentação geográfica e nacionalidade). Por isso, procurou-se avaliar a associação entre algumas variáveis, através do cruzamento de informações como sexo, faixa etária e frequência e formas de consumo (Tabela 2 e Tabela 3).

Analisando a Tabela 2, identifica-se que o consumo maior ocorre esporadicamente, independente do sexo e idade. Além disso, entre o público feminino o maior consumo nos finais de semana ocorre na faixa etária até 21 anos e de 22 a 30 anos, somando 12,50\%. Ainda considerando o público feminino, ressalta-se que o consumo diário ou mais de uma vez na semana é inferior comparado ao público masculino. Dentre os homens, destaca-se um consumo mais efetivo na faixa etária acima dos 31 anos e de forma esporádica $(25 \%)$.

Tabela 2 - Comportamento dos provadores sobre a frequência de consumo estratificados pelo sexo e faixa etária.

\begin{tabular}{|c|c|c|c|c|c|c|c|c|c|c|c|c|}
\hline \multirow[t]{2}{*}{ Variáveis } & \multicolumn{2}{|c|}{ Diariamente } & \multicolumn{2}{|c|}{$\begin{array}{l}\text { De } 1 \text { a } 2 \\
\text { vezes na } \\
\text { semana }\end{array}$} & \multicolumn{2}{|c|}{$\begin{array}{l}\text { De } 2 \text { a } 3 \\
\text { vezes na } \\
\text { semana }\end{array}$} & \multicolumn{2}{|c|}{$\begin{array}{c}\text { Finais de semana } \\
\text { ou ocasiões } \\
\text { especiais }\end{array}$} & \multicolumn{2}{|c|}{ Esporadicamente } & \multicolumn{2}{|r|}{ Total } \\
\hline & $\mathrm{N}$ & $\%$ & $\mathrm{~N}$ & $\%$ & $\mathrm{~N}$ & $\%$ & $\mathrm{~N}$ & $\%$ & $\mathrm{~N}$ & $\%$ & $\mathrm{~N}$ & $\%$ \\
\hline Feminino & 1 & $3,13 \%$ & & $0,00 \%$ & 1 & $3,13 \%$ & 6 & $18,75 \%$ & 9 & $28,13 \%$ & 17 & $53,13 \%$ \\
\hline Até 21 anos & - & - & - & - & - & - & 2 & $6,25 \%$ & - & - & 2 & $6,25 \%$ \\
\hline De 22 a 30 anos & 1 & $3,13 \%$ & - & - & 1 & $3,13 \%$ & 2 & $6,25 \%$ & 3 & $9,38 \%$ & 7 & $21,88 \%$ \\
\hline De 31 a 40 anos & - & - & - & - & - & - & 1 & $3,13 \%$ & 1 & $3,13 \%$ & 2 & $6,25 \%$ \\
\hline De 41 a 50 anos & - & - & - & - & - & - & 1 & $3,13 \%$ & 4 & $12,50 \%$ & 5 & $15,63 \%$ \\
\hline Mais de 51 anos & - & - & - & - & - & - & - & - & 1 & $3,13 \%$ & 1 & $3,13 \%$ \\
\hline Masculino & 1 & $3,13 \%$ & 2 & $6,25 \%$ & 2 & $6,25 \%$ & 2 & $6,25 \%$ & 8 & $25,00 \%$ & 15 & $46,88 \%$ \\
\hline De 22 a 30 anos & 1 & $3,13 \%$ & - & - & - & - & 1 & $3,13 \%$ & - & - & 2 & $6,25 \%$ \\
\hline De 31 a 40 anos & - & - & 1 & $3,13 \%$ & 1 & $3,13 \%$ & - & - & 3 & $9,38 \%$ & 5 & $15,63 \%$ \\
\hline De 41 a 50 anos & - & - & - & - & 1 & $3,13 \%$ & 1 & $3,13 \%$ & 1 & $3,13 \%$ & 3 & $9,38 \%$ \\
\hline Mais de 51 anos & - & - & 1 & $3,13 \%$ & - & - & - & - & 4 & $12,50 \%$ & 5 & $15,63 \%$ \\
\hline Total Geral & 2 & $6,25 \%$ & 2 & $6,25 \%$ & 3 & $9,38 \%$ & 8 & $25,00 \%$ & 17 & $53,13 \%$ & 32 & $100,00 \%$ \\
\hline
\end{tabular}

Legenda: $\mathrm{N}=$ número de participantes do estudo. Fonte: Autores.

Apesar de Rapacci (1997) afirmar que o requeijão é um produto bastante consumido pela população em geral e de Van Dender (2014) dizer que devido ao fato do requeijão apresentar geralmente sabor mais suave em relação aos queijos naturais e por isso está tendo maior aceitação do público jovem, no presente estudo identificou-se que o maior consumo de 
requeijão ocorreu, entre as mulheres na faixa de 22 a 30 anos (21,88\%), seguido da faixa etária de 41 a 50 anos (15,63\%). Dentre os homens, o que se observou foi que o maior consumo se deu nas faixas etárias de 31 a 40 anos e acima de 51 anos, ou seja, um público mais experiente.

Quando analisa-se o comportamento dos provadores em relação as formas de consumo levando em consideração o sexo e faixa etária, temos os dados demonstrados na Tabela 3.

Tabela 3 - Comportamento dos provadores sobre as formas de consumo estratificados pelo sexo e faixa etária.

\begin{tabular}{|c|c|c|c|c|c|c|}
\hline \multirow{2}{*}{ Variáveis } & \multicolumn{2}{|c|}{ Em pães e bolachas } & \multicolumn{2}{|c|}{ Receitas culinárias } & \multicolumn{2}{|c|}{ Total } \\
\hline & $\mathrm{N}$ & $\%$ & $\mathrm{~N}$ & $\%$ & $\mathrm{~N}$ & $\%$ \\
\hline Feminino & 5 & $15,63 \%$ & 12 & $37,50 \%$ & 17 & $53,13 \%$ \\
\hline Até 21 anos & 1 & $3,13 \%$ & 1 & $3,13 \%$ & 2 & $6,25 \%$ \\
\hline De 22 a 30 anos & 2 & $656,25 \%$ & 5 & $15,63 \%$ & 7 & $21,88 \%$ \\
\hline De 31 a 40 anos & 1 & $3,13 \%$ & 1 & $3,13 \%$ & 2 & $6,25 \%$ \\
\hline De 41 a 50 anos & 1 & $3,13 \%$ & 4 & $12,50 \%$ & 5 & $15,63 \%$ \\
\hline Mais de 51 anos & - & - & 1 & $3,13 \%$ & 1 & $3,13 \%$ \\
\hline Masculino & 4 & $12,50 \%$ & 11 & $34,38 \%$ & 15 & $46,88 \%$ \\
\hline De 22 a 30 anos & 2 & $6,25 \%$ & - & - & 2 & $6,25 \%$ \\
\hline De 31 a 40 anos & 1 & $3,13 \%$ & 4 & $12,50 \%$ & 5 & $15,63 \%$ \\
\hline De 41 a 50 anos & - & - & 3 & $9,38 \%$ & 3 & $9,38 \%$ \\
\hline Mais de 51 anos & 1 & $3,13 \%$ & 4 & $12,50 \%$ & 5 & $15,63 \%$ \\
\hline Total Geral & 9 & $28,13 \%$ & 23 & $\mathbf{7 1 , 8 8 \%}$ & 32 & $100,00 \%$ \\
\hline
\end{tabular}

Legenda: $\mathrm{N}$ = número de participantes do estudo. Fonte: Autores.

Neste caso, observa-se que as mulheres consomem e/ou utilizam mais o produto, tanto em pão e bolachas quanto em receitas culinárias. Muito provavelmente pelo fato destas serem as responsáveis pelo preparo das refeições. Além disso, identificou-se que o maior consumo em pães e bolachas ocorre pelos provadores da faixa etária de 22 a 30 anos (6,25\%). Da mesma forma, é este público que mais utiliza o produto em questão nas mais diferentes receitas culinárias (15,63\%). Cabe ressaltar aqui que as mulheres acima de 51 anos não demonstraram consumir o produto em pães, somente em receitas culinárias.

Ao analisarmos o comportamento masculino nas diferentes faixas etárias, percebe-se que, assim como as mulheres, o maior consumo em pães ocorre pela faixa etária de 22 a 30 anos (6,25\%). Já quem utiliza o produto em receitas culinárias, são os homens de 31 a 40 anos $(12,50 \%)$ e os com idade superior a 51 anos $(12,50 \%)$.

Visando avaliar a associação entre as variáveis qualitativas relacionadas aos atributos que fazem o consumidor gostar do produto, os dados foram estratificados pelo sexo e renda (Tabela 4). 
Tabela 4 - Comportamento dos provadores sobre os atributos que fazem o consumidor gostar do produto estratificados pelo sexo e renda.

\begin{tabular}{|c|c|c|c|c|c|c|c|c|c|c|}
\hline \multirow{2}{*}{ Variáveis } & \multicolumn{2}{|c|}{$\begin{array}{c}\text { Aspectos } \\
\text { Sensoriais }\end{array}$} & \multicolumn{2}{|c|}{$\begin{array}{l}\text { Benefícios } \\
\text { a saúde }\end{array}$} & \multicolumn{2}{|c|}{ Praticidade } & \multicolumn{2}{|r|}{ Preço } & \multicolumn{2}{|r|}{ Total } \\
\hline & $\mathrm{N}$ & $\%$ & $\mathrm{~N}$ & $\%$ & $\mathrm{~N}$ & $\%$ & $\mathrm{~N}$ & $\%$ & $\mathrm{~N}$ & $\%$ \\
\hline Feminino & 10 & $31,25 \%$ & 2 & $6,25 \%$ & & $0,00 \%$ & 5 & $15,63 \%$ & 17 & $53,13 \%$ \\
\hline Até 1 salário mínimo & - & - & 1 & $3,13 \%$ & - & - & - & - & 1 & $3,13 \%$ \\
\hline De 1 a 2 salários mínimos & 2 & $6,25 \%$ & 1 & $3,13 \%$ & - & - & 3 & $9,38 \%$ & 6 & $18,75 \%$ \\
\hline De 2 a 4 salários mínimos & 7 & $21,88 \%$ & - & - & - & - & 2 & $6,25 \%$ & 9 & $28,13 \%$ \\
\hline Mais de 4 salários mínimos & 1 & $3,13 \%$ & - & - & - & - & - & - & 1 & $3,13 \%$ \\
\hline Masculino & 10 & $31,25 \%$ & 2 & $6,25 \%$ & 1 & $3,13 \%$ & 2 & $6,25 \%$ & 15 & $46,88 \%$ \\
\hline Até 1 salário mínimo & - & - & - & - & - & - & - & - & - & - \\
\hline De 1 a 2 salários mínimos & 2 & $6,25 \%$ & 1 & $3,13 \%$ & - & - & 2 & $6,25 \%$ & 5 & $15,63 \%$ \\
\hline De 2 a 4 salários mínimos & 4 & $12,50 \%$ & 1 & $3,13 \%$ & 1 & $3,13 \%$ & - & - & 6 & $18,75 \%$ \\
\hline Mais de 4 salários mínimos & 4 & $12,50 \%$ & - & - & - & - & - & - & 4 & $12,50 \%$ \\
\hline Total Geral & 20 & $62,50 \%$ & 4 & $12,50 \%$ & 1 & $3,13 \%$ & 7 & $21,88 \%$ & 32 & $100,00 \%$ \\
\hline
\end{tabular}

Legenda: $\mathrm{N}$ = número de participantes do estudo. Fonte: Autores.

Pode-se observar que tanto o público feminino quando masculino levam em consideração, principalmente, os aspectos sensorias dos produtos (ambos com 31,25\%). Mulheres com a renda de um a quatro salários mínimos costumam analisar mais o preço ao comprar um produto em relação aos homens (15,63\% e 6,25\%, respectivamente). Rapacci (1997) cita a renda como uma das principais variáveis sobre a ingestão de lácteos, a qual definem o padrão de consumo. Segundo o autor citado, o aumento da renda gera um aumento do consumo. Este comportamento citado por Rapacci pôde ser observado no presente estudo. Contudo, o fenômeno ocorreu até os quatro salários mínimos, para ambos os sexos. Observou-se uma diminuição do consumo pelos provadores que ganham acima de quatro salários mínimos.

O teste de aceitabilidade é empregado para medir o grau de gostar ou não de um determinado produto, ou pela intensidade do prazer no consumo, através dos testes de classificação (Dutcosky, 2015). No presente estudo, na avaliação sensorial de aceitabilidade avaliou-se quatro atributos, sendo eles, a aparência do produto, o aroma, textura, e o sabor, conforme citam Damodaran \& Parkin (2019). De acordo com os autores citados as preferências alimentares dos seres humanos estão baseadas justamente nestes atributos, pois a interação entre os componentes do alimentos (proteínas, gorduras, etc) resultam nestes atributos, como por exemplo, as propriedades de coagulação e textura em produtos lácteos ocorre devido a estrutura coloidal das micelas de caseína.

Neste sentido, procurou-se avaliar a associação entre a variável qualitativa relacionada aos atributos sensoriais de textura, aparência, sabor e aroma através da estratificação dos dados em sexo e renda (Tabela 5). 
Tabela 5 - Conhecimento e comportamento dos provadores sobre os atributos sensoriais estratificados pelo sexo e renda.

\begin{tabular}{|c|c|c|c|c|c|c|c|c|c|c|c|}
\hline & \multirow{2}{*}{ Variáveis } & \multicolumn{2}{|c|}{ Gostei Muitíssimo } & \multicolumn{2}{|c|}{ Gostei Muito } & \multicolumn{2}{|c|}{ Gostei } & \multicolumn{2}{|c|}{ Gostei Pouco } & \multicolumn{2}{|r|}{ Total } \\
\hline & & $\mathrm{N}$ & $\%$ & $\mathrm{~N}$ & $\%$ & $\mathrm{~N}$ & $\%$ & $\mathrm{~N}$ & $\%$ & $\mathrm{~N}$ & $\%$ \\
\hline \multirow{10}{*}{ 苞 } & Feminino & 11 & $34,38 \%$ & 2 & $6,25 \%$ & 4 & $12,50 \%$ & - & - & 17 & $53,13 \%$ \\
\hline & Até 1 salário mínimo & 1 & $3,13 \%$ & - & - & - & - & - & - & 1 & $3,13 \%$ \\
\hline & De 1 a 2 salários mínimos & 2 & $6,25 \%$ & 1 & $3,13 \%$ & 3 & $9,38 \%$ & - & - & 6 & $18,75 \%$ \\
\hline & De 2 a 4 salários mínimos & 7 & $21,88 \%$ & 1 & $3,13 \%$ & 1 & $3,13 \%$ & - & - & 9 & $28,13 \%$ \\
\hline & Mais de 4 salários mínimos & 1 & $3,13 \%$ & - & - & - & - & - & - & 1 & $3,13 \%$ \\
\hline & Masculino & 7 & $21,88 \%$ & 7 & $\mathbf{2 1 , 8 8 \%}$ & 1 & $3,13 \%$ & - & - & 15 & $46,88 \%$ \\
\hline & De 1 a 2 salários mínimos & 3 & $9,38 \%$ & 2 & $6,25 \%$ & - & - & - & - & 5 & $15,63 \%$ \\
\hline & De 2 a 4 salários mínimos & 2 & $6,25 \%$ & 3 & $9,38 \%$ & 1 & $3,13 \%$ & - & - & 6 & $18,75 \%$ \\
\hline & Mais de 4 salários mínimos & 2 & $6,25 \%$ & 2 & $6,25 \%$ & - & - & - & - & 4 & $12,50 \%$ \\
\hline & Total Geral & 18 & $56,25 \%$ & 9 & $28,13 \%$ & 5 & $15,63 \%$ & - & - & 32 & $100,00 \%$ \\
\hline \multirow{9}{*}{$\begin{array}{l}\ddot{\circ} \\
\frac{0}{\tilde{n}}\end{array}$} & Feminino & 11 & $34,38 \%$ & 2 & $6,25 \%$ & 4 & $12,50 \%$ & - & - & 17 & $53,13 \%$ \\
\hline & Até 1 salário mínimo & 1 & $3,13 \%$ & - & - & - & - & - & - & 1 & $3,13 \%$ \\
\hline & De 1 a 2 salários mínimos & 2 & $6,25 \%$ & 1 & $3,13 \%$ & 3 & $9,38 \%$ & - & - & 6 & $18,75 \%$ \\
\hline & De 2 a 4 salários mínimos & 7 & $21,88 \%$ & 1 & $3,13 \%$ & 1 & $3,13 \%$ & - & - & 9 & $28,13 \%$ \\
\hline & Mais de 4 salários mínimos & 1 & $3,13 \%$ & - & - & - & - & - & - & 1 & $3,13 \%$ \\
\hline & Masculino & 7 & $21,88 \%$ & 7 & $21,88 \%$ & 1 & $3,13 \%$ & - & - & 15 & $46,88 \%$ \\
\hline & De 1 a 2 salários mínimos & 3 & $9,38 \%$ & 2 & $6,25 \%$ & - & - & - & - & 5 & $15,63 \%$ \\
\hline & De 2 a 4 salários mínimos & 2 & $6,25 \%$ & 3 & $9,38 \%$ & 1 & $3,13 \%$ & - & - & 6 & $18,75 \%$ \\
\hline & Mais de 4 salários mínimos & 2 & $6,25 \%$ & 2 & $6,25 \%$ & - & - & - & - & 4 & $12,50 \%$ \\
\hline \multirow{11}{*}{ 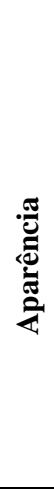 } & Total Geral & 18 & $56,25 \%$ & 9 & $28,13 \%$ & 5 & $15,63 \%$ & - & - & 32 & $100,00 \%$ \\
\hline & Feminino & 10 & $31,25 \%$ & 4 & $12,50 \%$ & 3 & $9,38 \%$ & - & - & 17 & $53,13 \%$ \\
\hline & Até 1 salário mínimo & - & - & 1 & $3,13 \%$ & - & - & - & - & 1 & $3,13 \%$ \\
\hline & De 1 a 2 salários mínimos & 2 & $6,25 \%$ & 2 & $6,25 \%$ & 2 & $6,25 \%$ & - & - & 6 & $18,75 \%$ \\
\hline & De 2 a 4 salários mínimos & 7 & $21,88 \%$ & 1 & $3,13 \%$ & 1 & $3,13 \%$ & - & - & 9 & $28,13 \%$ \\
\hline & Mais de 4 salários mínimos & 1 & $3,13 \%$ & - & - & - & - & - & - & 1 & $3,13 \%$ \\
\hline & Masculino & 4 & $12,50 \%$ & 10 & $31,25 \%$ & 1 & $3,13 \%$ & - & - & 15 & $46,88 \%$ \\
\hline & De 1 a 2 salários mínimos & 2 & $6,25 \%$ & 3 & $9,38 \%$ & - & - & - & - & 5 & $15,63 \%$ \\
\hline & De 2 a 4 salários mínimos & 1 & $3,13 \%$ & 5 & $15,63 \%$ & - & - & - & - & 6 & $18,75 \%$ \\
\hline & Mais de 4 salários mínimos & 1 & $3,13 \%$ & 2 & $6,25 \%$ & 1 & $3,13 \%$ & - & - & 4 & $12,50 \%$ \\
\hline & Total Geral & 14 & $43,75 \%$ & 14 & $43,75 \%$ & 4 & $12,50 \%$ & - & - & 32 & $100,00 \%$ \\
\hline \multirow{10}{*}{ 祸 } & Feminino & 8 & $25,00 \%$ & 4 & $12,50 \%$ & 3 & $\mathbf{9 , 3 8 \%}$ & 2 & $6,25 \%$ & 17 & $53,13 \%$ \\
\hline & Até 1 salário mínimo & - & - & 1 & $3,13 \%$ & - & - & - & $0,00 \%$ & 1 & $3,13 \%$ \\
\hline & De 1 a 2 salários mínimos & 2 & $6,25 \%$ & 1 & $3,13 \%$ & 2 & $6,25 \%$ & 1 & $3,13 \%$ & 6 & $18,75 \%$ \\
\hline & De 2 a 4 salários mínimos & 6 & $18,75 \%$ & 2 & $6,25 \%$ & - & - & 1 & $3,13 \%$ & 9 & $28,13 \%$ \\
\hline & Mais de 4 salários mínimos & - & - & - & - & 1 & $3,13 \%$ & - & $0,00 \%$ & 1 & $3,13 \%$ \\
\hline & Masculino & 6 & $18,75 \%$ & 7 & $\mathbf{2 1 , 8 8 \%}$ & 2 & $6,25 \%$ & & $0,00 \%$ & 15 & $46,88 \%$ \\
\hline & De 1 a 2 salários mínimos & 3 & $9,38 \%$ & 2 & $6,25 \%$ & & $0,00 \%$ & & $0,00 \%$ & 5 & $15,63 \%$ \\
\hline & De 2 a 4 salários mínimos & 2 & $6,25 \%$ & 4 & $12,50 \%$ & & $0,00 \%$ & & $0,00 \%$ & 6 & $18,75 \%$ \\
\hline & Mais de 4 salários mínimos & 1 & $3,13 \%$ & 1 & $3,13 \%$ & 2 & $6,25 \%$ & & $0,00 \%$ & 4 & $12,50 \%$ \\
\hline & Total Geral & 14 & $43,75 \%$ & 11 & $34,38 \%$ & 5 & $15,63 \%$ & 2 & $6,25 \%$ & 32 & $100,00 \%$ \\
\hline
\end{tabular}

Legenda: $\mathrm{N}$ = número de participantes do estudo. Fonte: Autores.

Analisando a Tabela 5 percebe-se claramente que as mulheres gostaram muitíssimo dos quatro atributos avaliados, principalmente aquelas com renda entre dois a quatro salários mínimos. Vale ressaltar o resultado apontado por duas provadoras que assinalaram ter gostado pouco do produto, o que representou 6,25\% do total de provadoras. Entre os homens, identificou-se que a preferência foi superior na faixa de renda de um a dois salários mínimos, sendo que eles sinalizaram que gostaram muitíssimo da textura $(21,88 \%)$ e sabor $(21,88 \%)$ e que gostaram muito da aparência $(31,25 \%)$ e do aroma $(21,88 \%)$. Silva, Amaral, Almeida, Braga e Damaceno (2021) também observaram que o gênero do consumidor influência os resultados. 
A aparência e a cor são atributos fundamentais na qualidade do produto, também são os primeiros a serem avaliados no momento da aquisição do produto pelos consumidores finais, que estão em busca de alimentos mais econômicos, seguros e nutritivos, sua aquisição não ocorrerá se estes não forem atraentes e nem chamarem a atenção. A cor também pode interferir na percepção do sabor, devido ao consumidor relacionar as cores com a qualidade do alimento, portanto torna essa uma característica decisiva na hora da escolha e aceitação do produto (Damodaran \& Parkin, 2019).

Por fim, os provadores foram questionados quanto a sua intenção de compra em relação ao produto desenvolvido e analisado (Figura 3). Conforme pode ser observado na Figura 3, 81\% disseram que certamente compraria e 19\% afirmaram que provavelmente comprariam, o que indica uma boa aceitação para o produto desenvolvido por parte dos provadores.

Figura 3 - Frequência relativa a intenção de compra do requeijão cremoso.

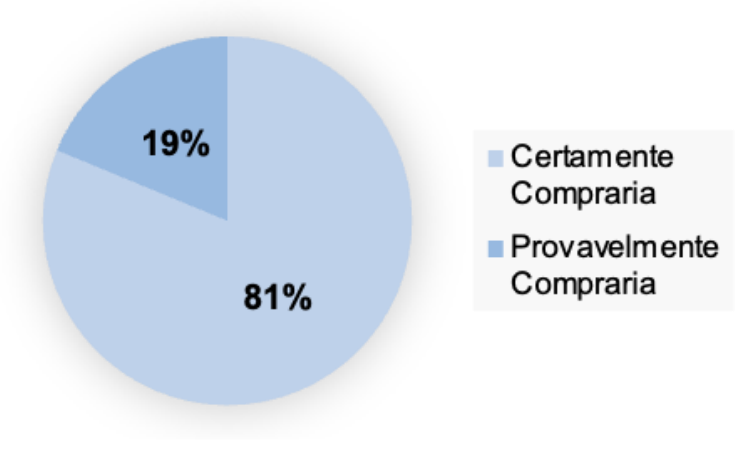

Fonte: Autores.

Salienta-se aqui o citado por Van Dender (2014) em relação ao sucesso e crescimento do consumo de requeijão. De acordo com o autor, o aumento na procura pelo produto de dá em função da ampla variedade de consistência, sabores, formas, funcionalidade (derretimento, espalhabilidade, fatiabilidade) e apelo ao consumidor.

\section{Conclusão}

A Análise sensorial demonstrou que o produto reformulado obteve boas avaliações em seus parâmetros sensoriais (aparência, aroma, textura e sabor).

Identificou-se que um possível perfil de consumidor de requeijão cremoso seriam as mulheres, na faixa etária de 22 a 40 anos, com ensino superior completo e renda de dois a quatro salários mínimos.

Como sugestão para trabalhos futuros, acredita-se que seria importante um estudo com uma população externa a empresa e com um número maior de participantes, com o objetivo de verificar se a perspectiva identificada neste estudo se confirma. Além disso, sugere-se um estudo de viabilidade econômica visando identificar se a nova formulação trará economia para a empresa.

\section{Referências}

Bald, J. A., Vicenzi, A., Gennari, A., Lehn, D. N., \& Souza, C. F. V. d. (2014). Características físico-químicas de soros de queijo e ricota produzidos no Vale do Taquari, RS. Revista Jovens Pesquisadores, 3(4), 96 - 100. doi: https://doi.org/10.17058/rjp.v4i3.4602

Bellarde, F. B. (2005). Elaboração de doce de leite pastoso com substituição parcial dos sólidos de leite por concentrado proteico de soro. Revista Brasileira Multidisciplinar, 2(9), 249 - 2056. doi: https://doi.org/10.25061/2527-2675/ReBraM/2006.v9i2.280

Cervo, A. L., Bervian, P. A., \& Silva, R. D. (2012). Metodologia científica. Pearson Prentice Hall.

Damodaran, S., \& Parkin, KL (2019). Química de alimentos de Fennema. Artmed.

Dutcosky, SD (2013). Análise sensorial de alimentos ( $4^{\mathrm{a}}$ ed.). Champagnat. 
Research, Society and Development, v. 11, n. 2, e41511225869, 2022

(CC BY 4.0) | ISSN 2525-3409 | DOI: http://dx.doi.org/10.33448/rsd-v11i2.25869

Fonseca, J. J. S. d. (2002). Metodologia da Pesquisa Científica. UECE. Retrieved January 17, 2022 from http://www.ia.ufrrj.br/ppgea/conteudo/conteudo2012-1/1SF/Sandra/apostilaMetodologia.pdf

Gil, A. C. (2021). Como elaborar projetos de pesquisa (6th ed.). Atlas.

Gigante, M. L. (1998). Requeijão cremoso obtido por ultrafiltração de leite pré-acidificado adicionado de concentrado proteico de soro [Tese de doutorado, Universidade Estadual de Campinas, Faculdade de Engenharia de Alimentos, Campinas]. BDTD. https://bdtd.ibict.br/vufind/Record/CAMP_71f27bbaeaf1fe4984c7ab27b52ed8db

Hoffmann, C.D. M. (2003). Estudo de utilização de concentrado proteico de soro de queijo ultrafiltrado (CPSU), em requeijão cremoso [Dissertação de Mestrado, Universidade Federal de Santa Catarina, Centro de Ciências Agrárias. Programa de Pós-Graduação em Ciência dos Alimentos.]. BDTD. https://bdtd.ibict.br/vufind/Record/UFSC_dd45ffbea991f6938b725a6c0a41c409

Lubeck, G. M. (2005). Estudo da fabricação de requeijão cremoso com diferentes concentrações de gordura no extrato seco, sal emulsificante e concentrado proteico de soro obtido por ultrafiltração [Tese de doutorado, Universidade Estadual de Campinas, Faculdade de Engenharia de Alimentos, Campinas]. BDTD. https://bdtd.ibict.br/vufind/Record/CAMP_3fe2e4eabadf4ebb614c98ba9cc4104d

Maia, G. P. A. G., Belisário, C. M., Silva, M. A. P. d., Oliveira, S. S., Loiola, C. M. d. F., \& Chagas, P. P. (2020, May 25). Soro de leite: vantagens da aplicação na formulação de alimentos. Milkpoint. https://www.milkpoint.com.br/artigos/industria-de-laticinios/vantagens-da-aplicacao-do-soro-de-leite-naformulacao-de-produtos-alimenticios-

219631/\#: :text=As\%20prote\%C3\%ADnas\%20presentes\%20no\%20soro,e\%20textura\%20agrad\%C3\%A1veis\%20ao\%20paladar

Marini, T. (2020). Desenvolvimento de iogurtes probióticos com elevado teor proteico por ultrafiltração [Dissertação de Mestrado, Ciência e Tecnologia de Alimentos, Instituto de Tecnologia de Alimentos]. ITAL. http://repositorio.ital.sp.gov.br/jspui/handle/123456789/107

Rapacci, M. (1997). Estudo comparativo das características físicas e químicas, reológicas e sensorias do requeijão cremoso obtido por estudo láctica e acidificação [Tese Doutorado, Universidade Estadual de Campinas, Faculdade de Engenharia de Alimentos, Campinas, SP.]. BDTD. http://www.repositorio.unicamp.br/handle/REPOSIP/255399

Sampieri, R. H., Collado, C. F., \& Lucio, M. d. P. B. (2013). Metodologia de Pesquisa (5th ed.). Penso.

Santos, G. (2017). Cálculo amostral: calculadora online. Prática Clínica. https://praticaclinica.com.br/anexos/ccolaborativa-calculo-amostral/ccolaborativacalculo-amostral.php

Siqueira, K., \& Schettino, JPJ (2021, 28 de abril). O consumo de queijos pelos brasileiros. Milkpoint. https://www.milkpoint.com.br/colunas/kennyasiqueira/o-consumo-de-queijos-pelos-brasileiros-225212/

Silva, F. S. d., Amaral, S. M. B., Almeida, A. P. F. d., Braga, R. C., \& Damaceno, M. N. (2021). Requeijão cremoso tradicional e light: aceitabilidade e correlação das medidas sensoriais e instrumentais. Research, Society and Development, 6(10), e30910615772. doi: https://doi.org/10.33448/rsd-v1Oi6.15772

Triviños, A. N. S. (1987). Introdução à pesquisa em ciências sociais: a pesquisa qualitativa em educação. Atlas.

Van Dender, AGF (2014). Requeijão cremoso e outros queijos fundidos (2 $2^{\mathrm{a}}$ ed.). Setembro Editora.

Zacarchenco, P. B., Van Dender, A. G. F., \& Rego, R. A. (Eds.). (2017). Brasil Dairy Trends 2020 (1st ed.). ITAL. Retrieved September 16, 2021 from http://brasildairytrends.com.br/ 